\title{
Monocyte Subsets, Stanford-A Acute Aortic Dissection, and Carotid Artery Stenosis: New Evidences
}

\author{
Noemi Cifani, ${ }^{1}$ Maria Proietta, ${ }^{2}$ Maurizio Taurino, ${ }^{3}$ Luigi Tritapepe $\left(\mathbb{D},{ }^{4}\right.$ \\ and Flavia Del Porto $\mathbb{D}^{5}$ \\ ${ }^{1}$ Dipartimento di Medicina Clinica e Molecolare, Ospedale Sant'Andrea, Facoltà di Medicina e Psicologia, Ospedale Sant'Andrea, \\ "Sapienza," Università di Roma, Italy \\ ${ }^{2}$ Dipartimento di Medicina Clinica e Molecolare, Ospedale Sant'Andrea, Facoltà di Medicina e Psicologia, Ospedale Sant'Andrea, \\ UOS Aterosclerosi e Dislipidemia, "Sapienza, " Università di Roma, Italy \\ ${ }^{3}$ Dipartimento di Medicina Clinica e Molecolare, Ospedale Sant'Andrea, Facoltà di Medicina e Psicologia, Ospedale Sant'Andrea, \\ UOC Chirurgia Vascolare, "Sapienza, " Università di Roma, Italy \\ ${ }^{4}$ Dipartimento di Scienze Anestesiologiche, Medicina Critica e Terapia del Dolore, Facoltà di Medicina e Odontoiatria, \\ Policlinico Umberto I, "Sapienza, " Università di Roma, Italy \\ ${ }^{5}$ Dipartimento di Medicina Clinica e Molecolare, Ospedale Sant'Andrea, Facoltà di Medicina e Psicologia, Ospedale Sant'Andrea, \\ UOC Medicina Interna, "Sapienza, "Università di Roma, Italy
}

Correspondence should be addressed to Flavia Del Porto; flavia.delporto@uniroma1.it

Received 21 February 2019; Revised 21 May 2019; Accepted 10 July 2019; Published 6 August 2019

Guest Editor: Mohammad A. Khan

Copyright (C) 2019 Noemi Cifani et al. This is an open access article distributed under the Creative Commons Attribution License, which permits unrestricted use, distribution, and reproduction in any medium, provided the original work is properly cited.

\begin{abstract}
Monocytes are a heterogeneous cell population distinguished into three subsets with distinctive phenotypic and functional properties: "classical" (CD14++CD16-), "intermediate" (CD14++CD16+), and "nonclassical" (CD14+CD16++). Monocyte subsets play a pivotal role in many inflammatory systemic diseases including atherosclerosis (ATS). Only a low number of studies evaluated monocyte behavior in patients affected by cardiovascular diseases, and data about their role in acute aortic dissection (AAD) are lacking. Thus, the aim of this study was to investigate CD14++CD16-, CD14++CD16+, and $\mathrm{CD} 14+\mathrm{CD} 16++$ cells in patients with Stanford-A AAD and in patients with carotid artery stenosis (CAS). Methods. 20 patients with carotid artery stenosis (CAS group), 17 patients with Stanford-A AAD (AAD group), and 17 subjects with traditional cardiovascular risk factors (RF group) were enrolled. Monocyte subset frequency was determined by flow cytometry. Results. Classical monocytes were significantly increased in the AAD group versus CAS and RF groups, whereas intermediate monocytes were significantly decreased in the AAD group versus CAS and RF groups. Conclusions. Results of this study identify in AAD patients a peculiar monocyte array that can partly explain depletion of T CD4+ lymphocyte subpopulations observed in patients affected by AAD.
\end{abstract}

\section{Introduction}

Atherosclerosis (ATS) is a multifactorial disease [1] characterized by an inflammatory remodeling of the arterial wall. Depending on size and site of vessels involved, ATS leads to a wide range of cardiovascular diseases (CVDs) [2], including ischemic heart disease, cerebrovascular disease, carotid artery stenosis (CAS), abdominal aortic aneurism (AAA), acute aortic dissection (AAD), and other conditions $[3,4]$. Immune response strongly affects the outcome of intraparietal inflammation: Thelper (Th) 1 lymphocytes have been mainly associated with plaque formation and Th2 lymphocytes with AAA, whereas macrophages have been related to $\operatorname{AAD}[1,5,6]$.

Monocytes represent the circulating precursor of tissue macrophages [7] and play an important role in atherogenesis, being rapidly attracted by activated endothelial cells [8]. During an atherosclerotic process, their differentiation into macrophages is associated with upregulation of phagocytic 
activity leading to lipid accumulation and formation of typical foam cells [1]. Monocytes are a heterogeneous cell population distinguished by the expression of the surface markers CD14 (coreceptor for LPS) and CD16 (receptor for Fc $\gamma$ RIII) [9] into three subsets: "classical" (CD14++CD16-), "intermediate" (CD14++CD16+), and "nonclassical" (CD14+ CD16++) [10]. Each monocyte subset possesses distinctive phenotypic and functional properties and displays different immune functions, distinguished by cytokine profiles and phagocytic activity [11]. A low number of studies evaluated monocyte behavior in patients affected by CVDs. Classical monocytes have been independently associated with cardiovascular events including death, myocardial infarction, and stroke [12, 13]. Furthermore, experimental evidences support the role of intermediate monocytes in atheroocclusive diseases [14], such as coronary artery disease (CAD) [15, 16], cardioembolic stroke [17], CAS [18], unstable angina [12, 18], and AAA [19]. However, to our knowledge, data about the role of monocyte subsets in AAD are still lacking.

Therefore, we evaluated CD14++CD16-, CD14++CD16+, and CD14+CD16++ cells in patients with Stanford-A AAD and in patients with CAS.

\section{Materials and Methods}

This was an observational retrospective study.

The population included in this study was composed of 17 patients undergoing Stanford-A AAD surgical repair at the Attilio Reale Heart and Great Vessels Department, Policlinico Umberto I, "Sapienza" University of Rome (AAD group). Patients were selected on the basis of the following inclusion criteria: (i) Stanford-A AAD; (ii) no history of neoplasm or autoimmune, infectious, or inflammatory systemic diseases; (iii) no presence of genetic syndromes known to be responsible for aortic disease; and (iv) no family history of aortic dissection or aneurysm.

A group of 20 patients with critical CAS (CAS group) was selected among those undergoing carotid thromboendo-arteriectomy (TEA) at the Department of Vascular Surgery, Sant'Andrea Hospital, "Sapienza" University of Rome. Patients were enrolled on the basis of the following inclusion criteria: (i) critical carotid stenosis, defined as a narrowing of the carotid lumen $\geq 70 \%[20,21]$; (ii) no cardiac causes of stroke; (iii) no history of neoplasm or autoimmune or inflammatory systemic diseases; and (iv) no familiar or personal history of aneurysms/dissection. All patients underwent physical and neurological examinations, carotid artery ultrasound, and angiography by magnetic resonance imaging (MRI) or contrast tomography (CT).

Seventeen patients with traditional cardiovascular risk factors attending the Department of Atherosclerosis and Dyslipidemia, Sant'Andrea Hospital, "Sapienza” University of Rome, were used as the control group (RF group). Patients were selected on the basis of the following criteria: (i) no acute cerebrovascular symptoms or history of cardiovascular disease, (ii) no carotid stenosis $>20 \%$, and (iii) no familiar or personal history of aneurysms/dissection.
No significant differences regarding age (mean age \pm SD: $68.83 \pm 4.11$ years, $59.85 \pm 11.01$ years, and $62.59 \pm$ 11.08 years for CAS, RF, and AAD, respectively), sex, diabetes, hypertension, dyslipidemia, and body mass index (BMI) were observed between CAS and RF groups.

AAD patients were matched with CAS and RF patients for age, sex, diabetes, and BMI but not for hypertension and dyslipidemia.

A venous blood sample was withdrawn from each patient (just before surgery) and from each control, in order to isolate peripheral blood mononuclear cells (PBMCs) by density gradient centrifugation (Lympholyte, Cedarlane, Hornby, CA). (Since Attilio Reale Heart and great Vessels Department is an hub reference center for $\mathrm{AAD}$, all patients underwent to surgery within 6 hours from the onset of the symptoms and blood samples were collected within this time).

Monocyte subsets were analyzed by flow cytometry as previously described [22-24] using the following antibodies: CD14 FITC (BD Biosciences, San Jose, CA, USA), CD16 APC (BD Biosciences, San Jose, CA, USA), and HLA-DR PE (BD Biosciences, San Jose, CA, USA). Briefly, cells were first visualized on FSC vs. SSC, and an ample gate was drawn around the monocyte cloud to exclude the majority of debris and lymphocytes. These cells were then viewed on a CD14 vs. CD16 plot. Moreover, the presence of natural killer (NK) cells, most of which are CD16-positive and could interfere with CD16+ monocyte count, was checked by HLA-DR antibody; accordingly, HLA-DR-negative NK cells were excluded. Monocyte subsets CD14+CD16-, CD14+CD16+, and $\mathrm{CD} 14+\mathrm{CD} 16++$ were, therefore, defined according to the surface expression of CD14 and CD16 [23,9] (Figure 1).

On the basis of the number of PBMC available, it was possible to test also CD4+ T lymphocytes in 6 patients of the CAS group, in 6 with RF, and in 10 with AAD. In the 10 AAD patients, immunohistochemistry of aortic specimens collected during surgery was performed as previously described [25].

FACS analysis was performed using a FACSCalibur cytometer (Becton-Dickinson) equipped with Cell Quest software. Isotype controls were used as compensation controls and to confirm antibody specificity.

All the statistical procedures were performed by GraphPad Prism 4 software (GraphPad Software Inc.).

The study was performed according to the principles of the Declaration of Helsinki and was approved by the ethics committee of the Faculty of Medicine.

Written informed consent was obtained from each patient or from an authorized family member.

\section{Results and Discussion}

\subsection{Results}

3.1.1. Monocytes. Classical monocytes were significantly increased in the AAD group versus CAS and RF groups $(p=0.0342$ and $p=0.0422$, respectively), whereas intermediate monocytes were significantly decreased in the AAD group versus CAS and RF groups $(p=0.0494$ and $p=$ 0.0211 , respectively). In particular, both intermediate and 


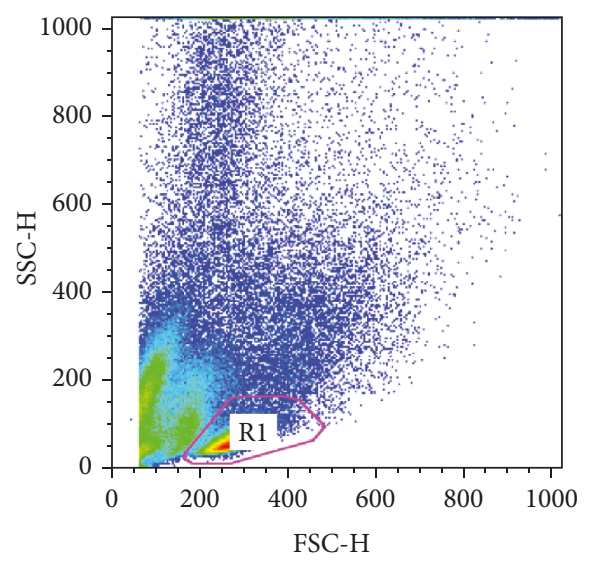

(a)

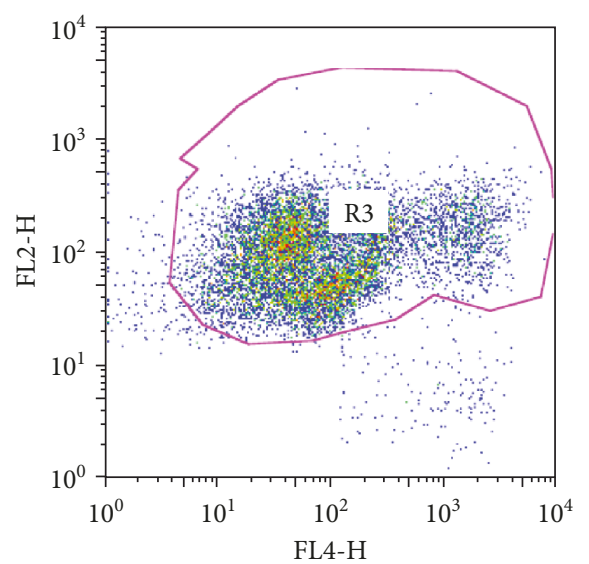

(c)

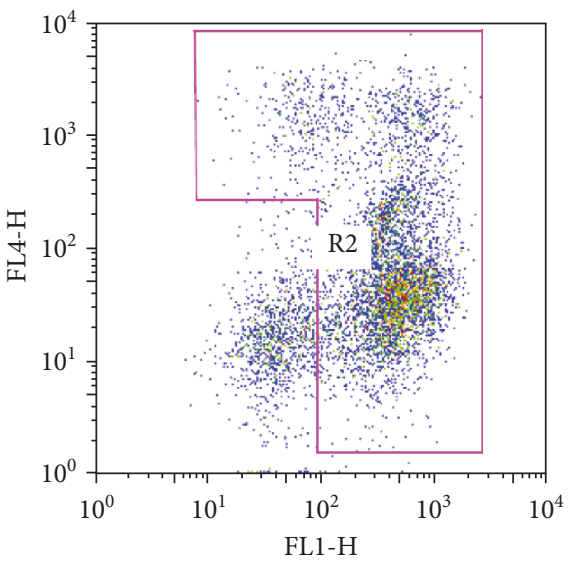

(b)

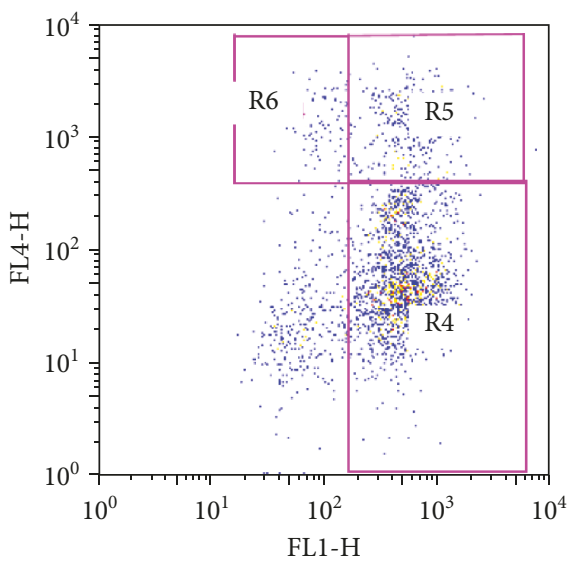

(d)

FIgURE 1: Representative flow cytometry strategy. Cells are visualized on FSC vs. SSC, and gate R1 is drawn around the monocyte cloud (a). These cells are then viewed on a CD14 (FITC, FL1-H) vs. CD16 (APC, FL4-H) plot, and gate R2 is drawn around the monocyte cloud (b). Gate R2 cells are viewed on a CD16 (APC, FL4-H) vs. HLA-DR (PE, FL2-H) plot, and HLA-DR-negative NK cells were excluded drawing R3 gate (c). Then, R3 monocyte population is viewed again on a CD14 (FITC, FL1-H) vs. CD16 (APC, FL4-H) plot, and CD14++CD16- (gate R4), CD14++CD16+ (gate R5), and CD14+CD16++ (gate R6) cells are defined according to the surface expression of CD14 and CD16 (d).

TABLE 1: Percentage of monocyte subsets in CAS, AAD, and RF groups.

\begin{tabular}{lccccc}
\hline & $\begin{array}{c}\text { Group CAS }(n=20) \\
\text { Mean } \pm \text { SD }\end{array}$ & $M$ & $\begin{array}{c}\text { Group AAD }(n=17) \\
\text { Mean } \pm \text { SD }\end{array}$ & M & $\begin{array}{c}\text { Group RF }(n=17) \\
\text { Mean } \pm \text { SD }\end{array}$ \\
\hline Classical monocytes & $93.05 \pm 4.21$ & 93.74 & $95.37 \pm 4.04$ & 97.11 & $91.11 \pm 7.68$ \\
Intermediate monocytes & $5.78 \pm 3.59$ & 5.45 & $3.69 \pm 3.23$ & 2.89 & $6.99 \pm 4.96$ \\
Nonclassical monocytes & $1.17 \pm 1.20$ & 0.65 & $0.94 \pm 1.19$ & 0.45 & $1.90 \pm 3.84$ \\
\hline
\end{tabular}

nonclassical monocytes progressively increased from AAD to $\mathrm{RF}$, although any significant difference was observed regarding the nonclassical subset (Table 1, Figure 2). No significant differences were observed between CAS and RF groups for all monocyte subsets.

3.1.2. Lymphocyte Subpopulations. A significant decrease of CD4+ T lymphocyte percentage $(p=0.05)$ was observed in $\mathrm{AAD}$ (mean $\pm \mathrm{SD}$ : $31.04 \pm 17.92$; median: 30.00$)$ versus CAS (mean \pm SD: $50.63 \pm 19.34$; median: 55.35 ). No signif- icant differences were observed between CAS and RF (mean \pm SD38.43 \pm 14.36 ; median 39.00) and between $\mathrm{AAD}$ and RF.

3.1.3. Immunohistochemistry. Data regarding immunohistochemistry are reported in Table 2. In 8/10 AAD samples, an inflammatory infiltrate was observed within the aortic wall. In $7 / 8$ samples, macrophages were the main population infiltrating the arterial wall, whereas only in one patient was observed a low infiltrate of T CD4+ lymphocytes. 

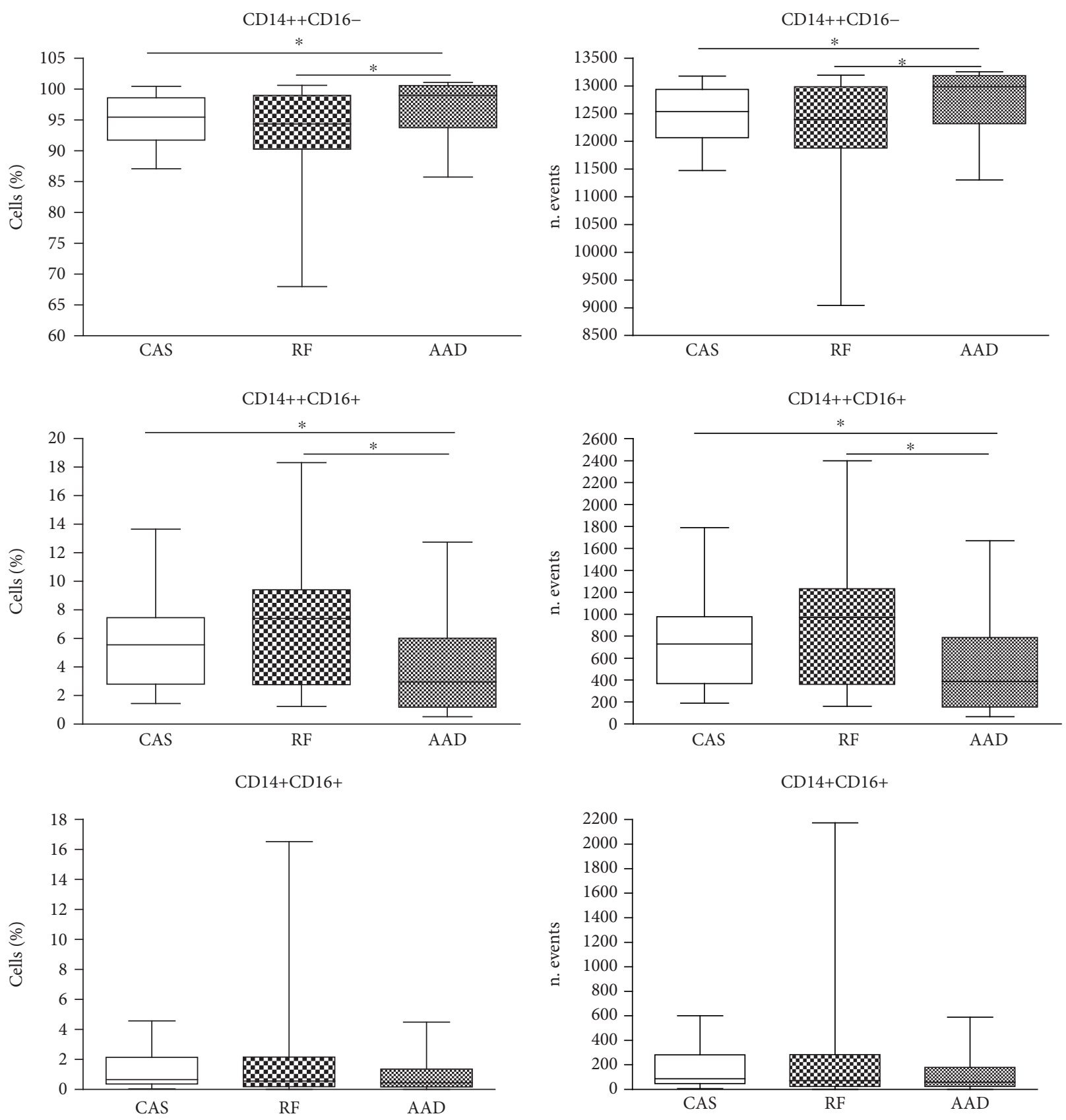

(a)

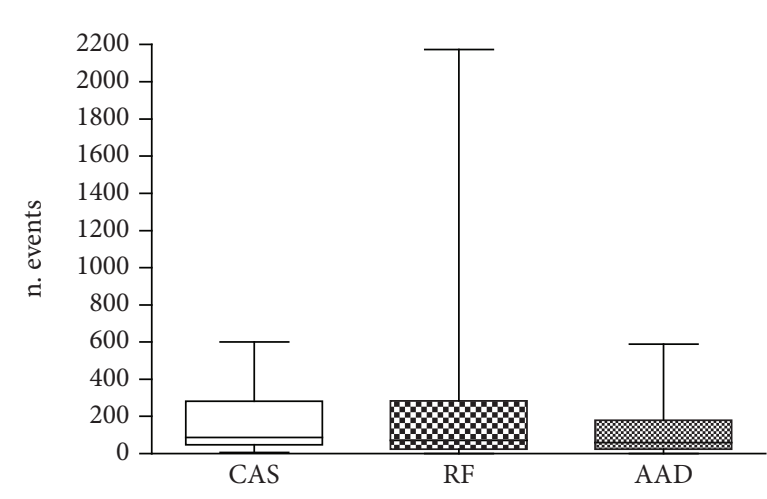

(b)

Figure 2: FACS analysis of monocyte subsets in CAS, RF, and AAD groups. Data were expressed as the percentage of cells (a) and the number of events (b). The 25 and 75 percentiles, median, minimal, and maximal are shown. Statistical analysis: Mann-Whitney nonparametric test. ${ }^{*} p<0.05$.

3.2. Discussion. Our results demonstrated that patients affected by AAD show a peculiar monocyte pattern characterized by elevated classic and reduced intermediate cell subsets, which predispose them to a prevalent natural immune response. Moreover, we observed that CAS and AAD patients displayed an opposite monocyte array, confirming that immune response plays a pivotal role in driving atherosclerotic parietal remodeling toward occlusion or rupture. In this field, it has been demonstrated that a prevalent CD4+ immune response directs subintimal inflammation toward plaque formation, whereas a prevalent innate macrophage activation underlies medial degeneration and aortic rupture in Stanford-A AAD patients with no genetic predisposition $[1,6]$.

We, indeed, considered $\mathrm{AAD}$ and asymptomatic critical CAS as the opposite sides of the same ATS diseases, in which immune response drives parietal remodeling toward rupture or stable occlusion. 
TABLE 2: Immune infiltrate within the aortic wall.

\begin{tabular}{lcc}
\hline Sample & Macrophages $(\mathrm{CD} 68+)$ & Lymphocytes $(\mathrm{CD} 4+)$ \\
\hline 1 & - & 1 \\
2 & 1 & 1 \\
3 & 1 & - \\
4 & - & - \\
5 & 3 & - \\
6 & - & - \\
7 & 2 & - \\
8 & 3 & 1 \\
9 & 3 & 2 \\
10 & 1 & - \\
\hline
\end{tabular}

Monocytes represent a systemic reservoir of myeloid precursors for renewal of tissue macrophages and dendritic cells, but they also exert effector/antigen-presenting cell and regulatory functions. Macrophages are the main cells involved in the innate immune response and play a crucial role in the inflammatory process underlying ATS [26]. These cells, indeed, express an array of inflammatory factors, as well as matrix metalloproteinases (MMPs), which are responsible for maintaining intraparietal inflammation and degrading extracellular matrix [27]. Their activation has been related to myocardial infarction, stroke, and CAS [28]. Moreover, several studies indicated, both in mice and in humans, that macrophage recall and their activation represent key events in the early phases of AAD [6].

Interestingly, monocytes are able to trigger and polarize $\mathrm{T}$ cell-mediated immune response [29-31]. In particular, intermediate monocytes exert proinflammatory actions $[10,32]$ and have been reported to favor $\mathrm{T}$ cell differentiation toward Th1 and Th17 [33].

Experimental evidences support the role of intermediate monocytes in atheroocclusive diseases [22-24], such as symptomatic and asymptomatic CAS, cardioembolic stroke, and unstable angina $[12,17,18]$. Our results confirmed a high percentage of intermediate monocytes in CAS patients, whereas such subset was decreased in the AAD group versus both CAS and RF. This suggests that such depletion is specifically related to aortic rupture and can at least in part explain the lack of $\mathrm{T}$ CD4+ subpopulations which characterizes Stanford-A AAD [6, 34]. We, indeed, confirm that CD4+ T lymphocytes are significantly reduced in peripheral blood of AAD patients in comparison with CAS [6]. Moreover, a prevalent macrophage infiltrate was found within the tunica media in aortic samples, whereas T CD4+ lymphocytes were poorly represented.

In the $\mathrm{AAD}$ group, a significant increase of classic monocytes was documented versus both CAS and RF. Monocyte CD14++CD16- are mainly involved in natural response against pathogens. Furthermore, this subset has been related to the inflammatory process occurring in ATS [35]. The increase of such pattern in Stanford-A AAD patients strongly confirms that inflammation underlies also ascending aortic wall rupture in patients with no genetic predisposition and supports the hypothesis of a microbial contribution to $\mathrm{AAD}[36]$.

\section{Conclusion}

This study seems of particular interest, since to our knowledge, it is the first report about monocyte subsets in AAD. We found that Stanford-A AAD patients with no genetic predisposition display a peculiar monocyte pattern, which strongly differs from that observed in the CAS group. We, therefore, can speculate that monocytes, particularly CD14++CD16+ cells, can represent the link between innate and adaptive immunity and can contribute to drive immune response toward a matrix degrading natural response.

\section{Data Availability}

The data used to support the findings of this study are available from the corresponding author upon request.

\section{Conflicts of Interest}

The authors have no conflict of interest including specific financial interest and relationships and affiliations relevant to the subject matter or materials discussed in the manuscript.

\section{Acknowledgments}

The authors thank Paola Del Porto for advice and support with flow cytometry and Cira Di Gioia for immunohistochemistry. This work was supported by the "Sapienza" University of Rome, financing for Research Project 2016.

\section{References}

[1] G. K. Hansson, "Inflammation, atherosclerosis, and coronary artery disease," The New England Journal of Medicine, vol. 352, no. 16, pp. 1685-1695, 2005.

[2] P. Zhong, D. Wu, X. Ye et al., "Secondary prevention of major cerebrovascular events with seven different statins: a multitreatment meta-analysis," Drug Design, Development and Therapy, vol. 11, pp. 2517-2526, 2017.

[3] N. Cifani, M. Proietta, L. Tritapepe et al., "Stanford-A acute aortic dissection, inflammation, and metalloproteinases: a review," Annals of Medicine, vol. 47, no. 6, pp. 441-446, 2015.

[4] N. D. Wong, "Epidemiology and prevention of cardiovascular disease," in Oxford Textbook of Global Public Health, R. Detels, M. Gulliford, Q. A. Karim, and C. C. Tan, Eds., Oxford University Press, 2015.

[5] E. M. Isselbacher, "Thoracic and abdominal aortic aneurysms," Circulation, vol. 111, no. 6, pp. 816-828, 2005.

[6] F. Del Porto, M. Proietta, L. Tritapepe et al., "Inflammation and immune response in acute aortic dissection," Annals of Medicine, vol. 42, no. 8, pp. 622-629, 2010.

[7] W. Wiktor-Jedrzejczakabd and S. Gordon, "Cytokine regulation of the macrophage (M phi) system studied using the colony stimulating factor-1-deficient op/op mouse," Physiological Reviews, vol. 76, no. 4, pp. 927-947, 1996. 
[8] J. Jongstra-Bilen, M. Haidari, S. N. Zhu, M. Chen, D. Guha, and M. I. Cybulsky, "Low-grade chronic inflammation in regions of the normal mouse arterial intima predisposed to atherosclerosis," The Journal of Experimental Medicine, vol. 203, no. 9, pp. 2073-2083, 2006.

[9] B. Passlick, D. Flieger, and H. W. Ziegler-Heitbrock, "Identification and characterization of a novel monocyte subpopulation in human peripheral blood," Blood, vol. 74, pp. 2527-2534, 1989.

[10] L. Ziegler-Heitbrock, P. Ancuta, S. Crowe et al., "Nomenclature of monocytes and dendritic cells in blood," Blood, vol. 116, no. 16, pp. e74-e80, 2010.

[11] E. Idzkowska, A. Eljaszewicz, P. Miklasz, W. J. Musial, A. M. Tycinska, and M. Moniuszko, "The role of different monocyte subsets in the pathogenesis of atherosclerosis and acute coronary syndromes," Scandinavian Journal of Immunology, vol. 82, no. 3, pp. 163-173, 2015.

[12] K. S. Rogacev, B. Cremers, A. M. Zawada et al., "CD14++CD16+ monocytes independently predict cardiovascular events: a cohort study of 951 patients referred for elective coronary angiography," Journal of the American College of Cardiology, vol. 60, no. 16, pp. 1512-1520, 2012.

[13] K. E. Berg, I. Ljungcrantz, L. Andersson et al., "Elevated CD 14++CD 16- monocytes predict cardiovascular events," Circulation: Cardiovascular Genetics, vol. 5, no. 1, pp. 122-131, 2012.

[14] K. A. Krychtiuk, S. P. Kastl, S. L. Hofbauer et al., "Monocyte subset distribution in patients with stable atherosclerosis and elevated levels of lipoprotein(a)," Journal of Clinical Lipidology, vol. 9, no. 4, pp. 533-541, 2015.

[15] K. A. Krychtiuk, S. P. Kastl, S. Pfaffenberger et al., "Association of small dense LDL serum levels and circulatingmonocyte subsets in stable coronary artery disease," PLoS One, vol. 10, no. 4, article e0123367, 2015.

[16] K. A. Krychtiuk, S. P. Kastl, S. Pfaffenberger et al., "Small high-density lipoprotein is associated with monocyte subsets in stable coronary artery disease," Atherosclerosis, vol. 237, no. 2, pp. 589-596, 2014.

[17] G. M. Grosse, W. J. Schulz-Schaeffer, O. E. Teebken et al., "Monocyte subsets and related chemokines in carotid artery stenosis and ischemic stroke," International Journal of Molecular Sciences, vol. 17, no. 4, p. 433, 2016.

[18] S. Zeng, X. Zhou, L. Ge et al., "Monocyte subsets and monocyte-platelet aggregates in patients with unstable angina," Journal of Thrombosis and Thrombolysis, vol. 38, no. 4, pp. 439-446, 2014.

[19] G. Ghigliotti, C. Barisione, S. Garibaldi et al., "CD16 monocyte subsets are increased in large abdominal aortic aneurysms and are differentially related with circulating and cell-associated biochemical and inflammatory biomarkers," Disease Markers, vol. 34, pp. 131-142, 2013.

[20] C. S. Kidwell and S. Warach, "Acute ischemic cerebrovascular syndrome. Diagnostic criteria,” Stroke, vol. 34, no. 12, pp. 2995-2998, 2003.

[21] G. Raman, D. Moorthy, N. Hadar et al., "Management strategies for asymptomatic carotid stenosis: a systematic review and meta-analysis," Annals of Internal Medicine, vol. 158, no. 9, pp. 676-685, 2013.

[22] R. D. Abeles, M. J. McPhail, D. Sowter et al., "CD14, CD16 and HLA-DR reliably identifies human monocytes and their subsets in the context of pathologically reduced HLA-DR expression by $\mathrm{CD} 14^{\mathrm{hi}} / \mathrm{CD} 16^{\text {neg }}$ monocytes: expansion of
$\mathrm{CD} 14^{\mathrm{hi}} / \mathrm{CD} 16^{\text {pos }}$ and contraction of $\mathrm{CD} 14^{\mathrm{lo}} / \mathrm{CD} 16^{\text {pos }}$ monocytes in acute liver failure," Cytometry Part A, vol. 81A, no. 10, pp. 823-834, 2012.

[23] L. Ziegler-Heitbrock and T. P. Hofer, "Toward a refined definition of monocyte subsets," Frontiers in Immunology, vol. 4, p. 23, 2013.

[24] G. P. Fadini, R. Cappellari, M. Mazzucato, C. Agostini, S. Vigili de Kreutzenberg, and A. Avogaro, "Monocyte-macrophage polarization balance in pre-diabetic individuals," Acta Diabetologica, vol. 50, no. 6, pp. 977-982, 2013.

[25] F. Del Porto, N. Cifani, M. Proietta et al., "Regulatory T CD4 + CD25+ lymphocytes increase in symptomatic carotid artery stenosis," Annals of Medicine, vol. 49, no. 4, pp. 283290, 2017.

[26] M. S. Elkind, P. Ramakrishnan, Y. P. Moon et al., "Infectious burden and risk of stroke: the northern Manhattan study," Archives of Neurology, vol. 67, no. 1, pp. 33-38, 2010.

[27] Z. S. Galis, G. K. Sukhova, R. Kranzhöfer, S. Clark, and P. Libby, "Macrophage foam cells from experimental atheroma constitutively produce matrix-degrading proteinases," Proceedings of the National Academy of Sciences of the United States of America, vol. 92, no. 2, pp. 402-406, 1995.

[28] O. M. Pello, C. Silvestre, M. De Pizzol, and V. Andrés, “A glimpse on the phenomenon of macrophage polarization during atherosclerosis," Immunobiology, vol. 216, no. 11, pp. 1172-1176, 2011.

[29] N. V. Serbina, T. Jia, T. M. Hohl, and E. G. Pamer, "Monocytemediated defense against microbial pathogens," Annual Review of Immunology, vol. 26, no. 1, pp. 421-452, 2008.

[30] H. G. Evans, N. J. Gullick, S. Kelly et al., "In vivo activated monocytes from the site of inflammation in humans specifically promote Th17 responses," Proceedings of the National Academy of Sciences of the United States of America, vol. 106, no. 15, pp. 6232-6237, 2009.

[31] I. Avraham-Davidi, S. Yona, M. Grunewald et al., "On-site education of VEGF-recruited monocytes improves their performance as angiogenic and arteriogenic accessory cells," Journal of Experimental Medicine, vol. 210, no. 12, pp. 26112625, 2013.

[32] M. Hristov and C. Weber, "Differential role of monocyte subsets in atherosclerosis," Thrombosis and Haemostasis, vol. 106, no. 11, pp. 757-762, 2011.

[33] H. Zhong, W. Bao, X. Li et al., "CD16+ monocytes control Tcell subset development in immune thrombocytopenia," Blood, vol. 120, no. 16, pp. 3326-3335, 2012.

[34] M. Proietta, L. Tritapepe, N. Cifani, L. Ferri, M. Taurino, and F. Del Porto, "MMP-12 as a new marker of Stanford-A acute aortic dissection," Annals of Medicine, vol. 46, no. 1, pp. 4448, 2014.

[35] J. Rojas, J. Salazar, M. S. Martínez et al., "Macrophage heterogeneity and plasticity: impact of macrophage biomarkers on atherosclerosis," Scientifica, vol. 2015, Article ID 851252, 17 pages, 2015.

[36] R. H. Mehta, R. Manfredini, F. Hassan et al., "Chronobiological patterns of acute aortic dissection," Circulation, vol. 106, no. 9, pp. 1110-1115, 2002. 


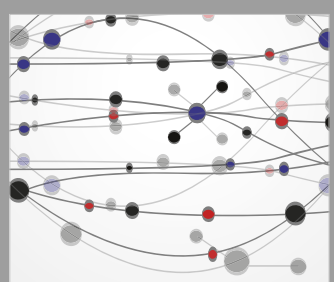

The Scientific World Journal
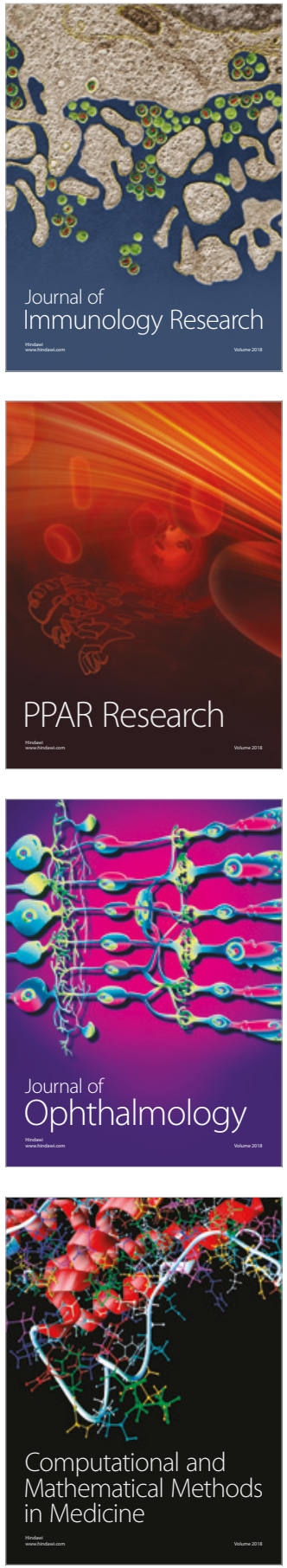

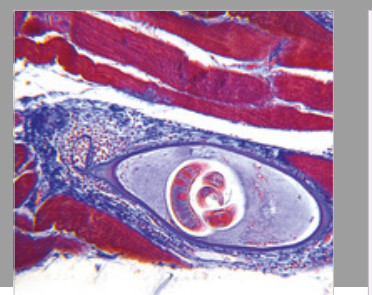

Gastroenterology Research and Practice

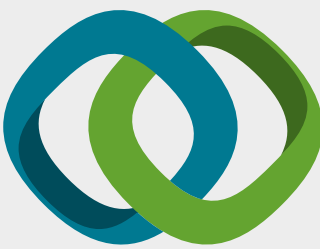

\section{Hindawi}

Submit your manuscripts at

www.hindawi.com
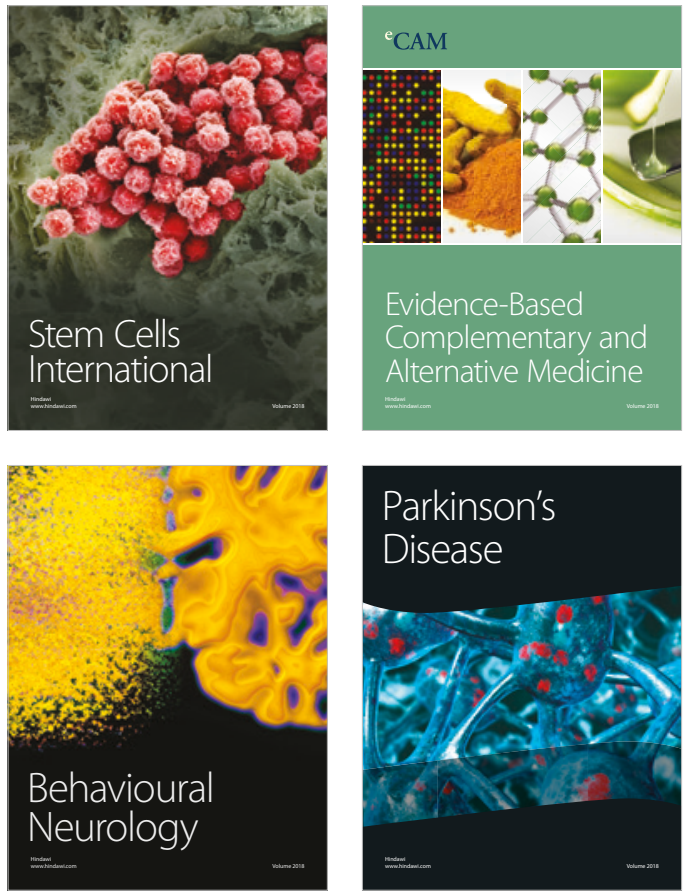

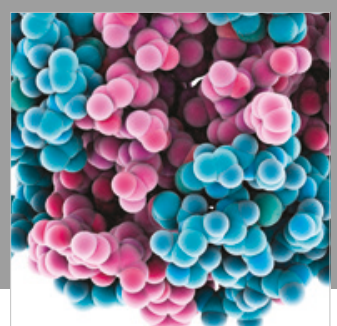

ournal of

Diabetes Research

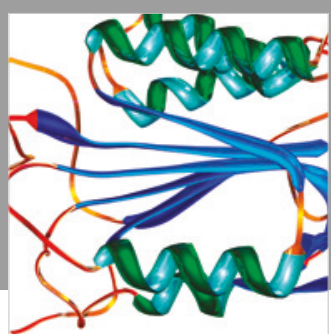

Disease Markers
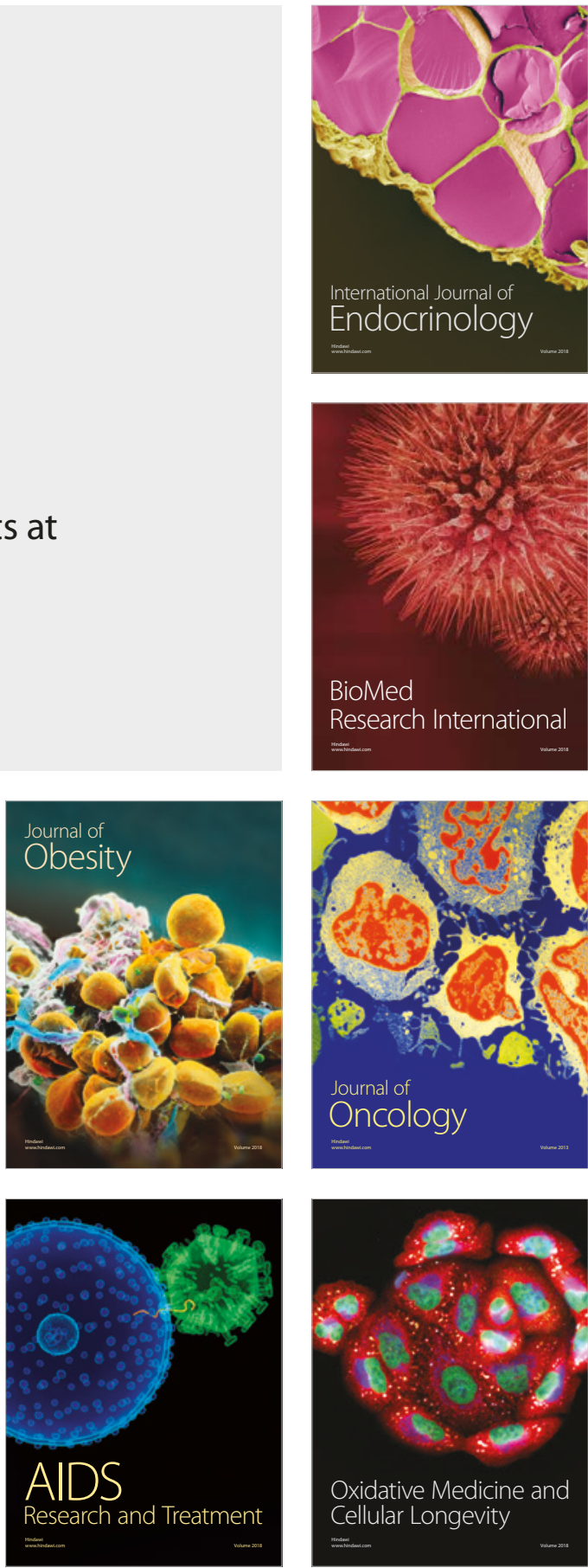